\title{
The Virtual Information Center Technologies for Open-Source Requirements (VICTOR) Project: Emerging HCI Concepts ${ }^{1}$
}

\author{
Ronald A. Moore and M. Gene Averett \\ Pacific Science \& Engineering Group \\ 6310 Greenwich Drive, Suite 200 \\ San Diego, CA 92122 \\ (858) 535-1661 \\ ramoore@pacific-science.com \\ averett@pacific-science.com
}

\author{
Jeff Clarkson \\ SPAWAR Systems Center, San Diego \\ Code D44206 \\ 53140 System Street \\ San Diego, CA 92152 \\ (619) 553-3534 \\ clarkson@spawar.navy.mil
}

\begin{abstract}
Regional and theater military operations have increasingly found open-source information to be a useful supplement to traditional data sources. Open-source information includes a wide variety of public data that can be readily acquired from news broadcasts, web sites, on-line databases, information brokers, email, etc. The VICTOR project is a technology demonstration initiative with the objective of developing human-centric decision support principles for information processing to support on-line open-source information analysis by leveraging existing / evolving capabilities. Observations, interviews, and product assessments were conducted at USCINCPAC to determine user requirements and current work processes. Based on this, we are developing a set of tools that are customized to address the cognitive tasks performed by open-source information analysts. An innovative development approach is being employed that provides access to a variety of relevant COTS/GOTS software through a consistent human-computer interface and that adapts these tools to the users' specific tasks. This approach enables extremely rapid, low cost development that stays abreast of the latest technology, while unburdening users from excessive system integration and training.
\end{abstract}

\section{Introduction}

Today's military environment requires timely and effective decision-making in a range of peacekeeping and war-fighting situations. To provide decision-makers with accurate information, analysts use a number of sophisticated tools and techniques to gather information from classified as well as open-sources. Open-source information includes a wide variety of publicly accessible data that can be acquired from news broadcasts, web sites, on-line databases, information brokers, email, etc. Unfortunately, many of the tools and techniques currently in use for this purpose are inadequate for military tasks, difficult to use, or lack the required level of integration and compatibility. Worse yet, some needed tools are technologically immature or simply do not exist. As a result, vital information is often inaccessible to the decision-maker.

\footnotetext{
${ }^{1}$ This work is sponsored by Dr. Michael Letsky, Office of Naval Research, in support of the Virtual Resource Center at USCINCPAC. The authors gratefully acknowledge assistance from Richard Kelly on an earlier draft.
} 


\section{Report Documentation Page}

Form Approved

OMB No. 0704-0188

Public reporting burden for the collection of information is estimated to average 1 hour per response, including the time for reviewing instructions, searching existing data sources, gathering and maintaining the data needed, and completing and reviewing the collection of information. Send comments regarding this burden estimate or any other aspect of this collection of information,

including suggestions for reducing this burden, to Washington Headquarters Services, Directorate for Information Operations and Reports, 1215 Jefferson Davis Highway, Suite 1204, Arlington

VA 22202-4302. Respondents should be aware that notwithstanding any other provision of law, no person shall be subject to a penalty for failing to comply with a collection of information if it

does not display a currently valid OMB control number.

1. REPORT DATE

2000

2. REPORT TYPE

3. DATES COVERED

00-00-2000 to 00-00-2000

4. TITLE AND SUBTITLE

The Virtual Information Center Technologies for Open-Source

Requirements (VICTOR) Project: Emerging HCI Concepts

6. AUTHOR(S)

7. PERFORMING ORGANIZATION NAME(S) AND ADDRESS(ES)

Pacific Science \& Engineering Group Inc,6310 Greenwich Drive Suite 200,San Diego,CA,92122

9. SPONSORING/MONITORING AGENCY NAME(S) AND ADDRESS(ES)

5a. CONTRACT NUMBER

5b. GRANT NUMBER

5c. PROGRAM ELEMENT NUMBER

5d. PROJECT NUMBER

5e. TASK NUMBER

5f. WORK UNIT NUMBER

8. PERFORMING ORGANIZATION

REPORT NUMBER

10. SPONSOR/MONITOR'S ACRONYM(S)

11. SPONSOR/MONITOR'S REPORT

$\operatorname{NUMBER}(\mathrm{S})$

12. DISTRIBUTION/AVAILABILITY STATEMENT

Approved for public release; distribution unlimited

13. SUPPLEMENTARY NOTES

The original document contains color images.

14. ABSTRACT

15. SUBJECT TERMS

16. SECURITY CLASSIFICATION OF:

a. REPORT

unclassified b. ABSTRACT

unclassified
17. LIMITATION OF ABSTRACT c. THIS PAGE

unclassified
18. NUMBER 19a. NAME OF

OF PAGES RESPONSIBLE PERSON

6 
SPAWAR Systems Center, San Diego (SSC-SD), under ONR sponsorship, is currently conducting the Virtual Information Center Technologies for Open-Source Requirements (VICTOR) Project to address these special information management requirements. Extensive efforts have been made to understand the user community and their needs, the tasks they perform, the resources and tools they use, and the products they generate. From this, we identified important issues and problem areas. A human-computer interface (HCI) design concept and demonstration were then developed highlighting a representative user's performance of routine tasks using the improved tools. Using the HCI design as a starting point, we have been developing a tool set to facilitate the identification, collection, fusion, analysis, management, and presentation of open-source data.

\section{User Requirements and Work Processes}

The primary customer of the VICTOR Project efforts is the Virtual Resource Center (VRC) located at USCINCPAC. Staffed by a group of highly skilled military and civilian researchers and analysts, the VRC collects and provides open-source information needed by US decision-makers. As the VICTOR tool set and related concepts mature, its products are intended to transition to other organizations with similar needs.

The first step in the human-centric decision support process used here is to understand the users' background, needs, tasks, resources, and products. To do this, we observed VRC personnel in situ, interviewed VRC staff about their tasks and resources, and analyzed the format and content of their projects. We also interviewed several "consumers" of VRC products to determine how well these products met their needs for open-source information.

\section{$2.1 \quad$ Open-Source Information Products}

The VRC generates several types of open-source information products. These include: (a) abstracts and summaries of relevant current news stories, (b) primers that compile a wide range of relevant information about a country or region, (c) research reports that predict long-term trends, (d) a database of frequently asked questions about the countries and regions within the Pacific theater, and (e) replies to specific requests for information from anyone in the command.

One dimension on which the VRC products differ is in the amount of situational context. Routine information searches occur when little context is available, while tailored searches occur in high context situations. Another dimension that characterizes VRC products concerns how they were initiated. Some products are reactive to requests for information from outside the VRC, while others are initiated by the VRC proactively.

Table 1 illustrates this representation of VRC products. Products in the first cell (routine reactive) result from implicit queries, where a standing request triggers the search on a regular basis. Products in the next cell (routine - proactive) emerge from the news stories reported each day. Tailored search products can be seen as arising from explicit queries (e.g., RFIs) or from an anticipation of decision-maker information needs (e.g., primers). 
Table 1. Representation of VRC Products

\begin{tabular}{|l||c|l|}
\hline & \multicolumn{1}{|c|}{$\begin{array}{c}\text { Routine Search } \\
\text { (Low Context) }\end{array}$} & \multicolumn{1}{c|}{$\begin{array}{c}\text { Tailored Search } \\
\text { (High Context) }\end{array}$} \\
\hline \hline Reactive & $\begin{array}{c}\text { Implicit Queries } \\
\text { e.g., Frequently Asked Questions }\end{array}$ & $\begin{array}{l}\text { Explicit Queries } \\
\text { e.g., Requests for Information }\end{array}$ \\
\hline Proactive & $\begin{array}{c}\text { Emergent Queries } \\
\text { e.g., News Abstracts / Summaries }\end{array}$ & $\begin{array}{l}\text { Anticipatory Queries } \\
\text { e.g., Primers, Research Reports }\end{array}$ \\
\hline
\end{tabular}

\section{$2.2 \quad$ VRC Staffing}

These products can be developed by several geographically distributed groups, linked together by an information network. This "virtual information center" includes the Center of Excellence - for connections with non-governmental and private volunteer organizations, the Pacific Disaster Center - for weather and oceanographic information, commercial information brokers - for indepth reports on selected topics, and the VRC at USCINCPAC.

Within the VRC, there are several military and civilian staff with varying degrees of experience with the open-source information analysis tasks. The Researchers, many of whom are reservists, use Internet search tools to gather basic open-source information such as news articles. The Analysts, then, use the information collected by the Researchers to build or adapt a product. The VRC Director oversees the work assignments of the Researchers and Analysts and reviews the final products before they are forwarded or posted. In addition, several software engineers and a system administrator support the VRC's operation.

\subsection{Work Processes and Cognitive Demands}

While generating the VRC products involves different resources and procedures, there are five general functions that are common to all: (a) structure the query / topic, (b) acquire the data, (c) organize the data, (d) interpret the data, and (e) produce the report. Work flow diagrams were created to characterize the detailed steps within each of these functions that are necessary to generate each type of VRC product, to identify resources and tools currently used, and to indicate how information is exchanged among staff.

Particular emphasis was focused on the tasks that were the most cognitively demanding and that continue to pose the greatest challenge for VRC staff. Two of these, structuring queries and summarizing retrieved data, are discussed in terms of their general requirements for the VICTOR tool set.

\subsubsection{Structuring Queries}

It is especially difficult to determine what topics and sources to search when a query for opensource information comes from someone outside the VRC. Queries phrased in natural language 
are easily and often misinterpreted, and the underlying goal of the request is not easy to discern. The VRC researcher / analyst must determine the context and purpose of the request, the intended use of the information, information that's already known, the deadline for the report, the precision required, etc. In addition, it is often helpful to get a starting place for the search, which sometimes may be a previous search, archived information, or even a good web site.

\subsubsection{Summarizing Information}

Following an Internet search on a topic, a huge number of web sites and articles are typically retrieved, many of which are conflicting or redundant. For example, in searching for information related to the query, "How many casualties might be expected following a major volcanic eruption in Indonesia?" we obtained over 330,000 potentially useful resource sites. Even though only a few hundred sites may be directly relevant, many of these are contradictory, uninformative, or not current. There is a need to summarize massive quantities of data into a single, concise answer. Summaries, however, should still indicate the quantity, reliability, location, and perishability of the data, should enable users to directly access the original data if desired, and should allow users to specify the amount of detail provided. To reduce the impact of decision biases, supporting, conflicting, missing, and related information should be presented together and significant assumptions, inferences, and "leaps of faith" should be made explicit.

\section{Prototype VICTOR Tools}

Several human-computer interface (HCI) functional requirements have emerged from this analysis. First, the VICTOR tool set must be easy to learn and easy to use. The large number of inexperienced, short-term staff, the high workload, and the rapid deadlines in the VRC make this requirement a high priority. Second, the VICTOR tool set must be powerful, taking advantage of state-of-the-art information technology in order to retrieve hard-to-find data without overwhelming users with redundant or irrelevant hits. Next, the tools must be flexible, since each mission is likely to require a different type of tools, methods, and data. Finally, the VICTOR tool set must be modular in order to stay abreast of the latest developments in the many related areas of information technology.

The prototype VICTOR tools are discussed in detail and demonstrated as part of this presentation. Figure 1 illustrates how these prototype tools relate to each other and fit with the VRC's work flow. 


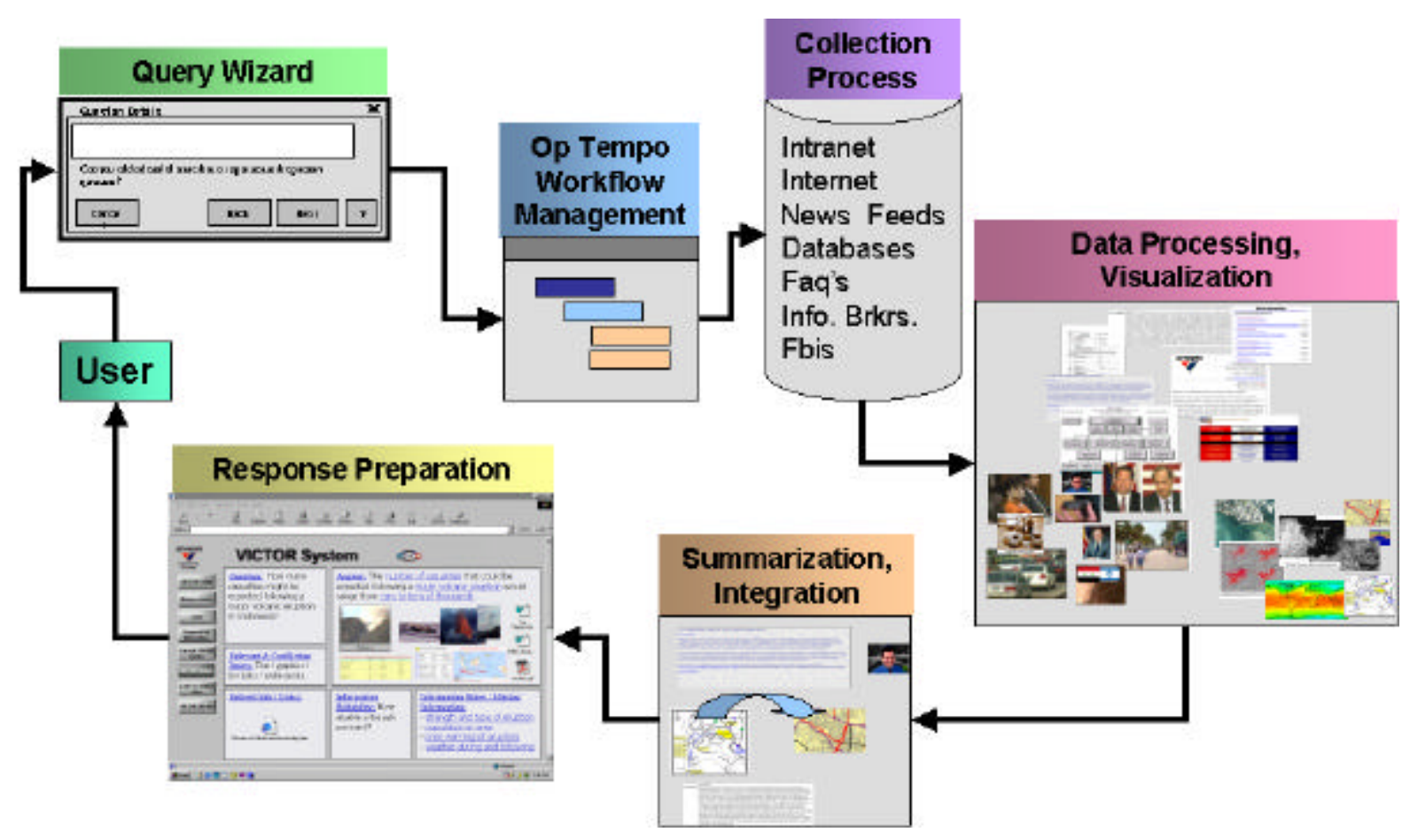

Figure 1. Prototype VICTOR tools that support open-source information analysis.

\subsection{Query Wizard}

A simple, interactive "interview agent" helps requestors and VRC analysts phrase and parse opensource information requests. This helps to disambiguate commonly used words, establish the context for the request, identify links to other requests, and determine the urgency of the request. The interview agent can also help construct multiple specific requests from a general query. For example, the general query, "What would be the effects of a major volcanic eruption in Indonesia?" might be parsed into separate queries focusing on the impact on the local population, the regional climate, the food supply, and the long-term economy.

\subsection{Workflow Management}

This tool helps the VRC staff to stay informed about upcoming events, task priorities, and status of ongoing analyses. It provides a useful focal point for updates and alerts about the latest world events and news that may be relevant to military concerns. The Director can also use this tool as a way to assign tasks to personnel, monitor workload of the staff, and to reset task priorities.

\subsection{Data Collection}

Many excellent search engines and agents are available commercially to support the VRC's need to collect open-source data on a wide range of topics. Rather than replicate any of these, this VICTOR tool provides users with a portal from which to access these commercial tools. However, the VICTOR Super Search tool provides a common HCI and makes it easy for users to 
specify the breadth, depth, and means of their searching. This tool is designed to serve the searching needs of both experts and novices.

\subsection{Data Visualization, Summarization, Integration, and Reporting}

VICTOR is developing a series of tools to help users to compile, organize, and visualize the diverse forms of open-source data that are typically retrieved. A key feature of this tool is its ability to present multi-media data in common workspaces to promote fusion and integration. As analysts' may require, other tools and simulations can be consulted via web links in order to help interpret, disambiguate, or forecast patterns in the retrieved data. Throughout this mediated analysis process, VICTOR is building a draft of the VRC report that fits structural templates customized for the kind of information needed by various types of open-source information consumers in the command.

\section{Leveraged Development}

Many of the VICTOR tools are intentionally similar to commercial software products that are in common usage at most offices, including the VRC. In fact, our design and development approach explicitly attempts to leverage these strengths to support our application. VICTOR tools provide a customized version of these applications, with pre-specified templates and links to other useful resources. There are many clear advantages to this leveraged development approach, including lower software development cost and time, quicker alignment with state-of-the-art commercial software applications, greater compatibility with tools used for other purposes, and reduced burden for training and system integration. The most significant challenge with this approach is to determine which applications best fit the user's task requirements and to develop an appropriate and usable common HCI from which users can access these applications. This is why the VICTOR project has adopted the human-centric decision support process that begins with a clear understanding of the users' tasks, products, resources, and workflow.

\section{Ongoing Efforts}

The VICTOR project is still in the early stages, but already several prototype technologies have been installed at the VRC in USCINCPAC for evaluation. Feedback from these prototypes has helped guide further development efforts to refine and expand the tools provided for open-source information analysis. Our particular focus over the next year will be on supporting the analysis and reporting of large amounts of conflicting open-source data using multi-media data visualization and conceptual structuring tools.

This spiral design-development-testing process has also been quite beneficial for the more basic research aspects of the project. By providing operational users with an initial version of some advanced information technology, we can determine whether and how they might actually use it and what pre- or post-processing may be required. This enables us to identify promising technologies rapidly, without a huge investment in software development. At the same time, this user feedback on prototypes has identified important unresolved questions where more basic research is needed through human factors, cognitive science, and team collaboration studies. 